\title{
A Weight Estimation Method for Prosthetic Hand
}

\author{
Bei Yang ${ }^{1,2}$, Xiaogang Duan ${ }^{1,2, *}$ and Hua Deng ${ }^{1,2}$ \\ ${ }^{1}$ State Key Laboratory of High-Performance Complex Manufacturing \\ ${ }^{2}$ School of Mechanical \& Electrical Engineering, Central South University, Changsha, 410083, China \\ Corresponding author
}

\begin{abstract}
To achieve a human like grasping with a multifingered robot hand, the grasping force should be controlled with using information from the grasped object such as its weight. In this paper, we propose a method for estimating the weight of a grasped object using the force output of Force Sensor Resistor (FSR) sensor. FSR sensor can measure the variation of grasping force when the disturbing force applied on the suface of the grasped object. These datas are analyzed by using the method of Fourier Transformation, and approximately estimate the weight of grasped object. Finally, we propose a method for controlling grasping force resist disturbing force applied to the grasped object using the estimated weight data.
\end{abstract}

Keywords-weight estimation; prosthetic hand; fourier transformation

\section{INTRODUCTION}

Fingers of human allow an object to be lifted using adequate grasping force and without slippage, even when the weight and friction coefficient of the object are unknown [1]. However, since each grasped object has a different coefficient of friction and weight, it is necessary to set the grasping force to the required value by estimating the weight of grasped object. Moreover, because of the limitation of the electromyography decoding technology, the weight of grasped object which estimating by human brain cannot be decoded and send to the prosthetic hand. Therefore, we propose a method which according to the the vibration frequency for estimating the weight of the grasped object, aims at providing a reference grasping force in the reflection control of the prosthetic hand.

In the last two decades, the research of the prosthetic hand grasping has drawn much attention in control engineering community. A part of the researchers focus on the grasping used by human hands, trying to find the method of setting grasping force to the required value. It is possible to grasp any object for which the weight and coefficient of friction is not known, at any speed by imitating the method of grasping objects used by humans [1-6]. As demonstrated in [1-3], methods for controlling the grasping force were proposed, some by increasing ratio of the tangential force or measuring the degree of eccentricity of the contact surface and some by detecting slip using force/torque sensors based on strain gauges. However, these methods can not provide a suitable force adding to the grasping force when the disturbing force applied on the grasped object. Then, some works [7-10] proposed methods for deciding a suitable grasping force based on Electromyography (EMG) signal. The EMG signal was a complicated signal, which can be uesd to set the grasping force to the required value when the disturbing force applied [7]. Moreover, the EMG signals from the body's intact musculature can be used to identify motion commands for the control of a prosthetic hand [8-9]. Nevertheless, there are many disadvantages in this method, EMG signal acquires noise while traveling through different tissues and the EMG detector collects signals from different motor units at a time which may generate interaction of different signals [10]. Overall, the information of weight which is acquired by the EMG signals is difficult to deliver to the prosthetic hand.

This paper shows an approach to estimate the weight of grasped object using FSR and method of calculation. According to the estimated weight, the optimal force can be added to the grasping force when the disturbing force applied. Beside, this method is invulnerable due to environmental vibrations and other signals. It is a remarkable fact that, the estimated weight is not absolutely accurate in the grasping process of human hand. Therefore, the method for weight estimation of prosthetic hand does not require high accuracy. Finally we carry out a series of experiments for the object even when the material and weight are different, by using the estimated method based on the output data of the FSR sensor.

\section{PROBLEM FORMULATION}

The prosthetic hand has a single DOF (Figure 1). The grasping process of prosthetic hand can be divided into two parts, the low-level control and high-level control.

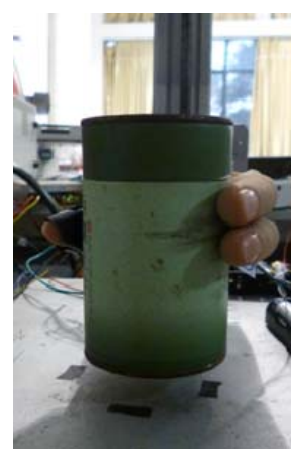

FIGURE I.

PROSTHETIC HAND

As shown in Figure 2, the desired force $F_{d}$ for prosthetic hand is formed from the decoding EMG signals, $\mathrm{C}$ denotes the controller, $\mathrm{P}$ is prosthetic hand, u denotes voltage signal and $F$ denotes the output of grasping force. There are many kinds of disturbance applied to grasped objects and the difficulties in the control process is summarized as follows,

1) It is hard to control the grasping force immediately when disturbing force applied to the grasped object and led to an unstable grasping.

2) An optimum force which added to the grasping force 
is hard to determine.

Thus, weight is an important parameter in adjusting the grasping force. A weight estimation method is presented in the following of the paper.

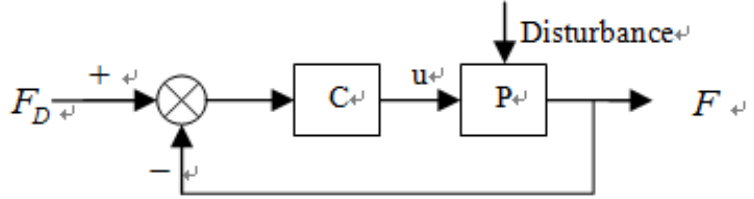

FIGURE II. THE CLOSED-LOOP SYSTEM OF PROSTHETIC HAND

III. PRinciple Of Weight ESTIMATION

The force analysis of graped object is illustrated in Figure 3. $F_{d}$ denotes the disturbing force, $F_{N}^{1}$ and $F_{N}^{2}$ are normal force exerted by fingers and $G$ is gravity.

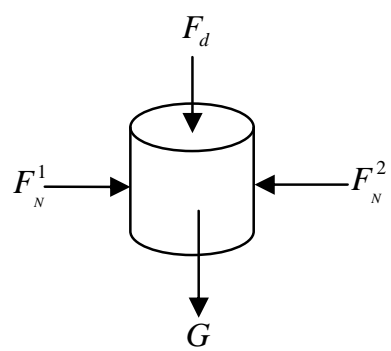

FIGURE III.

FORCE ANALYSIS OF GRASPED OBJECT

In view of the finger with Flexible glove, there exist elasticity and damping. Thus, using the Newton's second law, the dynamic model of the grasped object can be described by,

$$
m \ddot{x}+c \dot{x}+k x=U
$$

where $U=G+F_{d}-2 F_{f}, F_{f}$ is friction $c$ and $k$ are damping coefficient and spring stiffness of the object, respectively.

In this paper, we suppose that the finger is immobile and the system is underdamped. Besides, we mainly discuss impluse disturbing force and solve the equation (1),

$$
x(t)=\frac{U \omega_{n}}{\sqrt{1-\zeta^{2}}} e^{-\zeta \omega_{n} t} \sin \omega_{d} t
$$

where $\omega_{n}=\sqrt{k / m}, \quad \zeta=c / \omega_{n}, \omega_{d}=\omega_{n} \sqrt{1-\zeta^{2}}$.

According to $v=\dot{x}$, the relative speed between the finger and grasped object is,

$$
v(t)=A e^{-\zeta \omega_{n} t} \sin \left(\omega_{d} t+\beta\right)
$$

where $f_{0}=\left(G-2 F_{f}\right) / k, \varphi=\tan ^{-1} \frac{\sqrt{1-\zeta^{2}}}{\zeta}, A=\frac{\sqrt{f_{0}^{2}+p^{2}+2 f_{0} p \cos \varphi}}{m \sqrt{1-\zeta^{2}}}$,

$$
\beta=\sin ^{-1} \frac{f_{0}-p \sin \varphi}{\sqrt{f_{0}^{2}+p^{2}+2 f_{0} p \cos \varphi}} .
$$

Based on the Stribeck friction model [11], the dynamic behavior of the friction at low velocity is,

$$
F_{f}(v)=f+\mu v
$$

where $f=f_{c}+\left(f_{s}-f_{c}\right) e^{-\left(\mathrm{y} / v_{s}\right)^{2}}, f_{c}$ is the Coulomb level, $f_{s}$ is static friction force, $\mu$ and $v_{s}$ are friction coefficient and the Stribeck velocity, respectively.

For simplicity, a first order Taylor expansion is used to approximate this model because it is nonlinear,

$$
F_{f}(v)=F_{f}\left(v_{0}\right)+\frac{d F_{f}\left(v_{0}\right)}{d v}\left(v-v_{0}\right)
$$

where $v_{0} \in\left(0, v_{s}\right)$. It can be rewritten as

$$
F_{f}(v)=f_{m}+n v
$$

where $f_{m}=F_{f}\left(v_{0}\right)-\left(-\frac{2\left(F_{s}-F_{c}\right) v_{0}^{2}}{v_{s}^{2}} e^{-\left(v_{0} / v_{s}\right)^{2}}+\mu\right) v_{0}$,

$$
n=-\frac{2\left(F_{s}-F_{c}\right) v_{0}^{2}}{v_{s}^{2}} e^{-\left(v_{0} / v_{s}\right)^{2}}+\mu
$$

Combine (3) and (6),

$$
F_{f}(t)=f_{m}+n A e^{-\zeta \omega_{n} t} \sin \left(\omega_{d} t+\beta\right)
$$

The relation between friction force and positive pressure is as following

$$
F_{f}=\mu \cdot F_{N}
$$

where $\mu$ is the coefficient of friction between prosthetic finger and object, $F_{N}$ is the grasping force.

We supposing that coefficient of kinetic friction $\mu$ is unchanged, basing on equation (7) and (8), the grasping force $F_{N}$ is

$$
F_{N}=\frac{f_{m}}{\mu}+\frac{n}{\mu} A e^{-\zeta \omega_{n} t} \sin \left(\omega_{d} t+\beta\right)
$$

From equation (9) we can know that the curve of grasped force is damped oscillation curve after a disturbing force applying to the object. As shown in Figure 2, there is a sudden change of grasping force when the object begining to slip. With the passage of time, the vibration of the grasping force attenuated and tended to be a steady value eventual. 
According to equation (9), driving force of motor and disturbing force can not change the frequency of vibration, only influence on the amplitude and phase. Thus, we can take advantage of this, to estimate the weight of grasped object. Firstly, estimate the equivalent the coefficient of elasticity $k_{e}$ between grasped objects and prosthetic hands using force sensor resistor (FSR) and angular displacement sensors.

After that, we use Fast Fourier Transformation (FFT) to estimate the vibration frequency $v$ of grasping force. Based on Discrete Fourier Transform (DFT), the calculating formula is,

$$
x(k)= \begin{cases}\sum_{n=0}^{N-1} x(n) W_{N}^{k n} & 0 \leq k \leq N-1 \\ 0 & \text { others }\end{cases}
$$

where $W_{N}=e^{-j \frac{2 \pi}{N}}=\cos (2 \pi / N)-j \sin (2 \pi / N)$, N stands for the length of input vector. In order to facilitate the FFT operations, let $N=2^{m}$.

From the result of FFT, we can see that the lowest lowest amplitude occurs at the frequency of $83.3 \mathrm{~Hz}$, i.e. $v=83.3 \mathrm{~Hz}$. Then the vibration period $T$ is obtained based on equation as follows,

$$
T=\frac{1}{v}
$$

The natural frequency of second-order system can be describe as

$$
\omega_{d}=\omega_{n} \sqrt{1-\zeta^{2}}
$$

where $\omega_{n}$ is natural frequency with zero damping, $\zeta$ is damping ratio.

$$
\omega_{d}=2 \pi / T
$$

where $T$ is vibration period.

Supposing the system of prosthetic hand is no damping, the natural frequency is

$$
\omega_{n} \approx \omega_{d}=\sqrt{k_{e} / m}
$$

where $k_{e}$ is the coefficient of elasticity between grasped objects and prosthetic hands.

Besides, the value of $k_{e}$ is obtained by siffness test, as shown in Table 1. Basing on equation (13) and (14), the approximate weight of an object is

$$
m \approx \frac{k_{e} T^{2}}{2 \pi^{2}}
$$

\section{A. Experimental Apparatus}

As shown in Figure 4, the experimental apparatus is mainly composed by the disturbance generator, three-fingered prosthetic hand, FSR, data acquisition and grasped object. A disturbance generator is used for imitating disturbance such as impluse disturbing force. The prosthetic hand is actuated by a DC motor (series 2657). A FSR (standard 402 sensor) mounted on the surface of thumb is used to capture force variations during the slip of grasped object. In order to collect the signal of sensors in the reflex control system, we use the NI PXIe6363 multifunction data acquisition card as data acquisition module and the sampling period is $1 \mathrm{~ms}$.

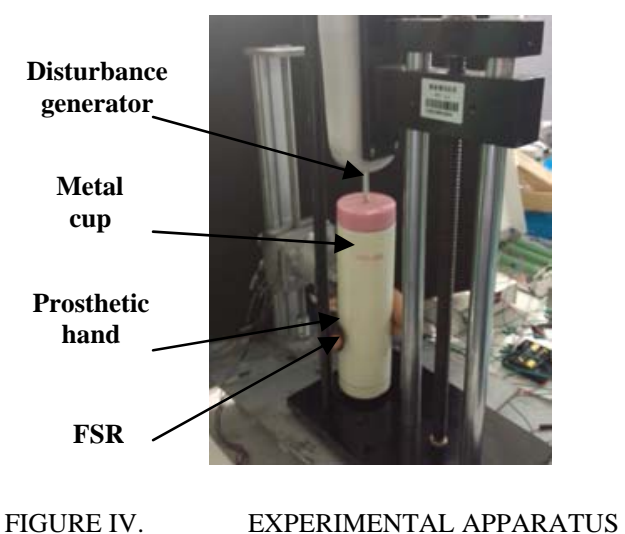

\section{B. Test Method}

In our approach, we carried out a series of experiments to verify the method. Experiment procedures are described as follows:

An object is grasped by prosthetic hand stably and kept it at a constant height.

The disturbance generator produces a certain disturbance that exerts on the top of the grasp object and the grasped object slips.

The variables of the experiments are the hardness of the object, the grasping force, disturbing force and weight. Therefore, we use different force to grasp hard, medium hardness, soft objects with different weight. The whole experiment divided into 9 groups based on different grasping forces and disturbing forces and each of these groups can be further subcategorized into 12 groups, the average values of datas obtained from each repeat tests category were used in this paper, the detail information is shown in Tabel 1. Futhermore, the experiments will repeat 3 times with the same grasping force under different disturbing force, e.g. the grasping forces of the experiment number $1,2,3$ are under the disturbing forces $1 \mathrm{~N}, 2 \mathrm{~N}, 4 \mathrm{~N}$ with the same grasping force $1 \mathrm{~N}$.

\section{RESUlTS AND DisCUSSIONS}

We can get the the vibration frequency by the method of FFT in each experimental result, then estimate the weight of the grasped object, as shown in Figure 5-7.

As shown in Table 2, the average values and the variances of estimated qualities were calculated from the quation below 
TABLE I. DETAILED PARAMETERS OF EXPERIMENTS

\begin{tabular}{|c|c|c|c|c|}
\hline Material & 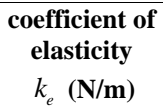 & $\begin{array}{l}\text { Experiment } \\
\text { group }\end{array}$ & $\begin{array}{c}\text { Actual } \\
\text { weight } \\
\text { (kg) }\end{array}$ & $\begin{array}{l}\text { Grasping } \\
\text { force(N) }\end{array}$ \\
\hline \multirow{3}{*}{ metal } & \multirow{3}{*}{107117} & $\mathrm{a}$ & 0.420 & $1 ; 2 ; 4 ; 6$ \\
\hline & & b & 0.620 & $2 ; 4 ; 6 ; 8$ \\
\hline & & C & 0.820 & $4 ; 6 ; 8 ; 10$ \\
\hline \multirow{3}{*}{ plastic } & \multirow{3}{*}{38343} & $\mathrm{~d}$ & 0.235 & $1 ; 2 ; 3 ; 4$ \\
\hline & & $\mathrm{e}$ & 0.435 & $2 ; 3 ; 4 ; 5$ \\
\hline & & $\mathrm{f}$ & 0.635 & $3 ; 4 ; 5 ; 6$ \\
\hline \multirow{3}{*}{ paper } & \multirow{3}{*}{9738} & g & 0.105 & $0.5 ; 1 ; 1.5 ; 2$ \\
\hline & & $\mathrm{h}$ & 0.265 & $1 ; 1.5 ; 2 ; 2.5$ \\
\hline & & $\mathrm{i}$ & 0.315 & $1.5 ; 2 ; 2.5 ; 3$ \\
\hline
\end{tabular}

$$
\begin{gathered}
\bar{m}=\frac{\sum m_{i}}{12} \\
\sigma^{2}=\frac{\sum_{i=1}^{12}\left(m_{i}-\bar{m}\right)^{2}}{12}
\end{gathered}
$$

where $i$ is experiment number, $i=1,2, \ldots, 12$

Besides, the absolute error and the relative error were defined as

$$
\begin{aligned}
& \Delta=|m-\bar{m}| \\
& \delta=(\Delta / m) \times 100 \%
\end{aligned}
$$

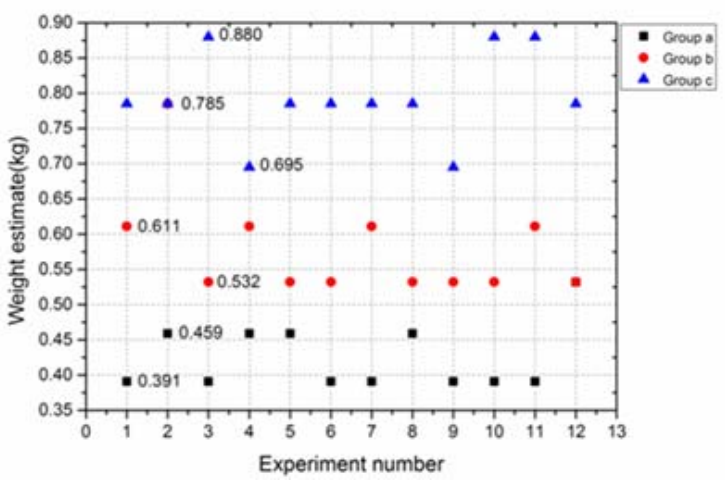

FIGURE V. THE ESTIMATED WEIGHT OF METAL CUP

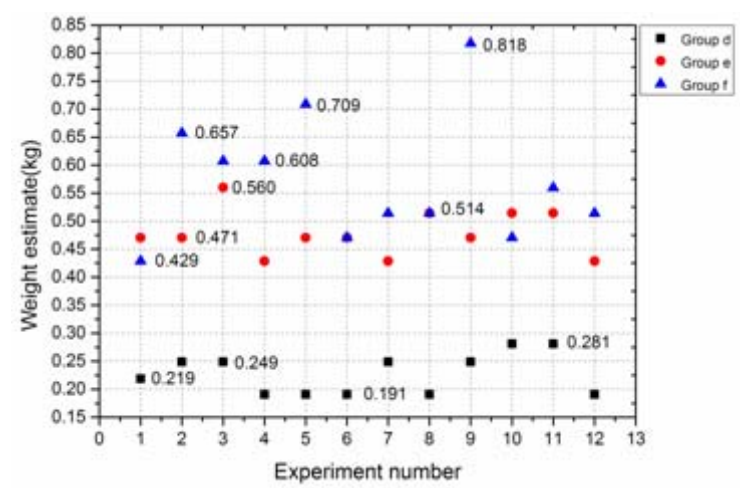

FIGURE VI.

THE ESTIMATED WEIGHT OF PLASTIC CUP

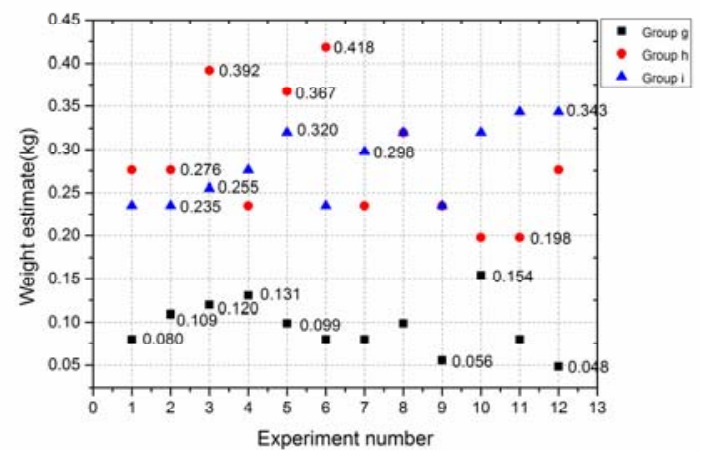

FIGURE VII.

THE ESTIMATED WEIGHT OF PAPER CUP

The specific calculation results are shown in Table 2 . According to the results, the variances of estimated qualities are no greater than 0.02 , the absolute error are less than $0.07 \mathrm{~kg}$ and the relative error are no greater than $10 \%$.

We can come to a conclusion that this method for estimating the weight of grasped object is feasible and the estimation accuracy can meet the requirement. Moreover, we demonstrated a method that the estimated weight can be used as a reference force which added to the grasping force in the closed-loop system when disturbing force applied on the grasped object.

TABLE II. RESULT OF WEIGHT ESTEMATION EXPERIMENTS

\begin{tabular}{|c|c|c|c|c|c|c|}
\hline \multirow{2}{*}{$\begin{array}{c}\text { Grasped } \\
\text { object }\end{array}$} & Group & \multirow{2}{*}{$\begin{array}{c}m_{0} \\
(\mathrm{~kg})\end{array}$} & $\begin{array}{c}\overline{\mathrm{m}} \\
\mathrm{kg})\end{array}$ & $\sigma^{2}$ & $\begin{array}{c}\Delta \\
(\mathrm{kg})\end{array}$ & $\begin{array}{c}\delta \\
(\%)\end{array}$ \\
\hline \multirow{3}{*}{ metal } & $\mathrm{a}$ & 0.420 & 0.426 & 0.0020 & 0.006 & 1.31 \\
\cline { 3 - 7 } & $\mathrm{b}$ & 0.620 & 0.584 & 0.0052 & 0.036 & 5.81 \\
\cline { 2 - 7 } & $\mathrm{C}$ & 0.820 & 0.794 & 0.0035 & 0.026 & 3.20 \\
\hline \multirow{3}{*}{ plastic } & $\mathrm{d}$ & 0.235 & 0.227 & 0.0012 & 0.008 & 3.23 \\
\cline { 2 - 7 } & $\mathrm{e}$ & 0.435 & 0.478 & 0.0015 & 0.043 & 1.00 \\
\cline { 2 - 7 } & $\mathrm{f}$ & 0.635 & 0.573 & 0.0120 & 0.062 & 9.82 \\
\hline \multirow{3}{*}{ paper } & $\mathrm{g}$ & 0.105 & 0.095 & 0.0090 & 0.010 & 9.93 \\
\cline { 2 - 7 } & $\mathrm{h}$ & 0.265 & 0.285 & 0.0050 & 0.020 & 7.72 \\
\cline { 2 - 7 } & $\mathrm{i}$ & 0.315 & 0.285 & 0.0018 & 0.030 & 9.64 \\
\hline
\end{tabular}

Note: $m_{0}$ is the actual weight of grasped object, $\bar{m}$ is the average value of estimated weight, $\sigma^{2}$ is variance, $\delta$ is absolute error relative error.

\section{CONCLUSION AND FUTURE WORK}

In this paper we proposed a weight estimat method using these grasping force output changes, and confirmed the effectiveness of the proposed method through testing. Furthermore, we proposed a method of adjusting grasping force in response to slip detected using sensor force output, and intesting using the proposed method.

This paper describes tests with a relatively simple disturbing force, but the proposed estimate method can probably also be applied to more complex disturbing force such as continuous disturbing force. Therefore in future we will examine estimate methods that take disturbing force into consideration, with the aim of achieving dexterous grasping similar to that of humans. 


\section{ACKNOWLEDGMENT}

In this paper, the research was sponsored by the grants from National Basic Research Program 973 of China (Grant No.2011CB013302).

\section{REFERENCES}

[1] T.Maeno, S.Hiromitsu, T.Kawai, "Control of Grasping Force by Estimating Stick/Slip Distribution at the Contact Interface of an Elastic Finger Having Curved Surface”, Journal of the Robotics Society of Japan, vol.19, no.1, pp.91-99,2001.

[2] A.Ikeda, Y.Kurita, J.Ueda, Y.Matsumoto, T.Ogasawara, "Grip Force Control of the Elastic Body based on Contact Surface Eccentricity During the Incipient Slip", Journal of the Robotics Society of Japan, vol.23, no.3, pp.337-343,2005.

[3] Claudio Melchiorri, "Slip Detection and Control Using Tactile and Force Sensors”, IEEE/ASME Transactionon Mechatronis, vol.5, no.3, pp.235243,2000 .

[4] T.Kawai, Y.Hirano, T.Maeno, "Development of Strain Distribution Sensor Having Curved Surface for Grip Force Control", The Japan Society of Mechanical Engineers. C, vol.64, No.627, pp.4264-4270, 1998.

[5] R.S.Johansson, G.Westling, "Roles of glabrous skin receptors and sensorimotor memory in automatic control of precision grip when lifting rougher or more slippery objects”, ExpBrainRes, vol.56, pp.550-564, 1984.

[6] R.S.Johansson, G.Westling, "Signals in tactile afferents from the fingers eliciting adaptive motor responses during precision grip”, Exp Brain Res, vol.66, pp.141-154, 1987.

[7] M. B. I. Reaz,M. S. Hussain and F. Mohd-Yasin Techniques of EMG signal analysis: detection, processing, classification and applications Journal () 1997-2006 Biological Procedures Online.

[8] Tucker C A and Liu X 1999 Self-organizing maps for time series analysis of electromyographic data IEEE Neural Netw. 5 3577-80.

[9] Christodoulou C I and Pattichis C S 1999 Unsupervised pattern recognition for the classification of EMG signals IEEE Trans. Biomed. Eng. 46 169-78.

[10] Shahid S. Higher Order Statistics TechniquesApplied to EMG Signal Analysis and Characterization. Ph.D. thesis, University of Limerick; Ireland, 2004.

[11] E. D. Engeberg, S. G. Meek, and M. A. Minor, "Hybrid Force-Velocity Sliding Mode Control of a Prosthetic Hand," IEEE Transactions on Biomedical Engineering, vol. 55, no. 5, pp. 1572-1581, 2008.

[12] S. H. Kang, M. Jin \& P. H. Chang, "A solution to the accuracy/robustness dilemma in impedance control", IEEE/ASME Transactions on Mechatronics, vol. 14, no. 3, pp. 282-294, 2009. 